\title{
Etude de la filière viande de chèvre au Zimbabwe
}

\author{
J. Gauthier ${ }^{1 *}$ A. Pradier ${ }^{2}$ F. Lecroisey ${ }^{2}$ C. Shumba ${ }^{3}$
}

\section{Mots-clés}

Viande caprine - Circuit de commercialisation - Economie agricole Modèle - Zimbabwe.

\section{Résumé}

Cet article présente une étude du secteur caprin au Zimbabwe, et plus particulièrcment dans la province de Masvingo, basée sur l'utilisation dú concept de filière comme outil d'analyse. Après une brève justification du choix analytique et une présentation des outils méthodologiques retenus, deux représentations de la filière sont proposées. Le deuxième modèle, plus puissant, est ensuite commenté au vu des données comptables. En conclusion, ce type d'analyse permet d'identifier des interlocuteurs privilégiés pour la mise en place d'actions de développement visant au renforcement des circuits de commercialisation de la viande de chèvre' et à l'amćlioration des revenus des petits producteurs.

\section{- INTRODUCTION}

Autrefois appelée Rhodésie du Sud, puis Rhodésie, le Zimbabwe a acquis son indépendance en 1980 (figure 1). D'une superficie de $390000 \mathrm{~km}^{2}$, ce pays compte environ 11 millions d'habitants dont 97,6 p. 100 sont d'origine africainc, 2 p. 100 d'origine curopéenne et 0,4 p. 100 asiatique.

Le régime foncier est encore marqué par l'ancien régime colonial. On distingue quatre types d'exploitation :

- les grandes fermes commerciales (zones commerciales) : au nombre de 4500 , elles sont exploitées par des propriétaires blancs et développent une agriculture moderne. D'une superficie moyenne de 2500 hectares, elles couvrent 40 p. 100 des terres agricoles ;

- les petites fermes commerciales : d'une superficie moyenne de 124 hectares, elles n'occupent que 4 p. 100 des terres agricoles et correspondent à d'anciennes grandes exploitations achetées par des Africains. Leur nombre, autour de 8600 , est resté constant au cours des dix dernières années ;

- les zones communales : couvrant 42 p. 100 des surfaces agricoles, ces zones comprennent des petites exploitations de quelques hectares gérées par des fermiers noirs ;

1. CIRAD-EMVT, LRVZ, BP 433, Ndjamena, Tchad

2. CIRAD-EMVT/Agritex, Box 354, Masvingo, Zimbabwe

3. Agritex, Box 354, Masvingo, Zimbabwe

* Auteur pour la correspondance
- les zones de réinstallation : nées de la politique de redistribution des terres, elles comprennent des exploitations légèrement plus grandes qu'en zones communales (en moyenne 5 hectares) et surtout situées dans des zones moins dégradées.

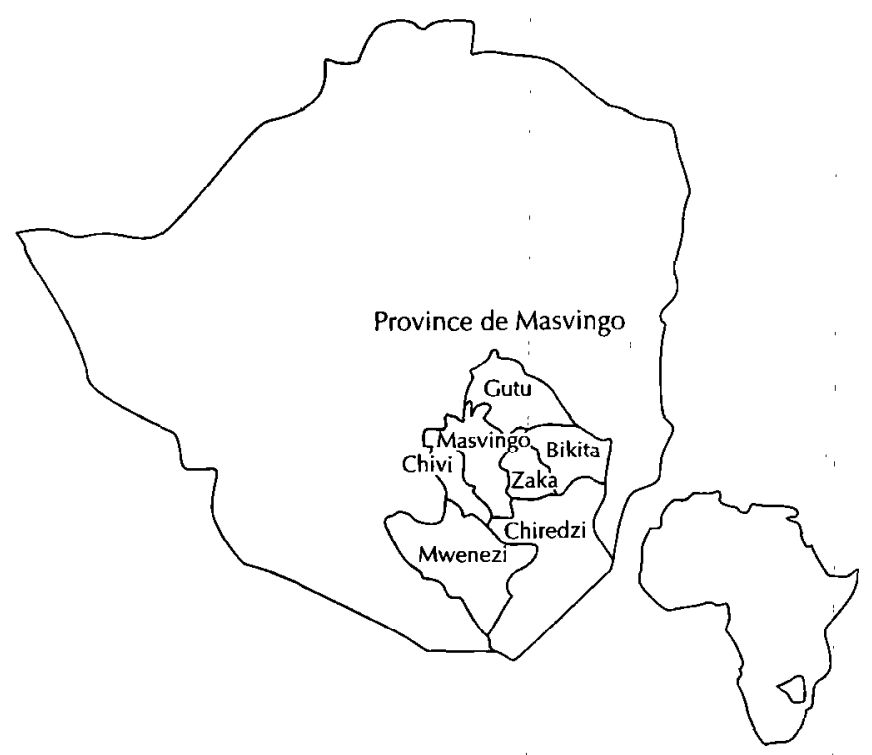

Figure 1 : le Zimbabwe, la province de Masvingo et ses districts. 
Dans ces différents systèmes fonciers, la chèvre a une importance variable. Très marginale dans les grandes fermes commerciales, la chèvre entre de façon plus marquée dans les systèmes d'élevage présents sur les autres systèmes fonciers. En moyenne, 90 p. 100 des exploitations possèdent des caprins. La part de la chèvre dans l'économie de ces fermes doit cependant être nuancée. Si l'on raisonne par rapport au revenu agricole obtenu par la vente de récoltes ou d'animaux, les chèvres vendues ne représentent que 12 , 2,2 et 1,7 p. 100 de ce revenu, respectivement dans les zones communales, de réinstallation et de petites fermes commerciales de la province de Masvingo. Les bovins, en revanche, représentent respectivement 27, 17 et 37 p. 100 de ce même revenu.

Dans le cadre d'un projet d'aide aux productions caprines dans la province de Masvingo (2), le département d'Elevage et médecine vétérinaire du Centre de coopération internationale en recherche agronomique pour le développement (CIRAD-EMVT) a été amené à travailler sur les circuits de commercialisation de la viande de chèvre. L'approvisionnement des villes restait insatisfaisant alors que les producteurs se plaignaient des difficultés de vente. D'où venait cette inadéquation entre l'offre et la demande ? A cette question aux conséquences théoriques s'ajoutait une question plus concrète : comment exprimer le potentiel connu de croissance de la production en viande caprine qui ne se faisait pas par manque de circuits de commercialisation?

Pour mener à bien ce travail, le concept de filière a été utilisé comme outil d'analyse (11). Dans cet article, après avoir rappelé brièvement les raisons de ce choix analytique, les auteurs se sont efforcés de montrer comment les différents éléments méthodologiques utilisés permettent de dépasser la simple représentation ensembliste que l'on peut avoir des différents acteurs de la filière pour obtenir une véritable compréhension des modes de fonctionnement et d'organisation de la filière, permettant des actions de développement plus pertinentes.

\section{METHODOLOGIE}

\section{Les limites du modèle néoclassique}

Le secteur caprin, qui fait l'objet de l'étude, aurait pu être approché avec les outils du modèle néoclassique standard développant une approche individualiste tout en faisant référence au marché et à son signal prix. Mais, comme le notifiait Agritex (service de vulgarisation et d'aide technique du Ministère zimbabwéen de l'Agriculture), notre partenaire institutionnel, il semble y avoir inadéquation entre l'offre et la demande en produits caprins au Zimbabwe, sans que le marché puisse jouer son rôle de régulateur. Plusieurs explications peuvent être avancées pour expliquer ce phénomène.

Cette inadéquation peut tout d'abord être liée à un problème de qualité, faisant que les acheteurs et les vendeurs ne peuvent pas s'entendre. Si l'on se réfère au poids de carcasse, il est vrai que les petites chèvres d'Afrique de l'Est ne fournissent que de petites carcasses rendant leur exploitation moins rentable pour les abatteurs. L'étude du comportement des abatteurs et des bouchers montre cependant que, s'ils recherchent plutôt des animaux plus lourds, ils se contentent actuellement des produits disponibles sur le marché.

Elle peut être liée aussi à une barrière d'entrée telle que des coûts de transport trop élevés, conséquence d'une trop grande distance entre les bassins de production et de consommation. Mais cette possibilité ne se justifie pas dans le cas de circuits locaux : bassins de production - villes limitrophes.
Enfin, elle peut être due à un manque d'organisation et donc à un coût de l'information trop élevé à certaines étapes du processus de production de la viande de chèvre. Ce troisième point a amené les auteurs à vouloir prendre en considération les modes de structuration et de fonctionnement des agents impliqués dans ce secteur d'activité.

\section{Les apports de la mésoanalyse}

\section{Le concept de filière}

Cherchant à mieux connaître les différents acteurs, leurs liens et leur comportement, les auteurs ont donc été amenés à dépasser le modèle micro-économique classique et à développer une approche mésoanalytique, basée sur un découpage de l'économie en « mésosystèmes dynamiques regroupant des agents liés par leurs relations marchandes et non marchandes plus fortes qu'avec le reste du monde » $(16,17)$.

La filière est un mésosystème particulier, basé sur une logique de produit, qui doit donc permettre de comprendre la structure et le fonctionnement des circuits commerciaux de la viande de chèvre.

Il n'existe cependant pas de méthode standard d'analyse de filière. Malgré un fort développement depuis les années 70 de ce type d'approche en France, le concept reste flou. En 1988, Soufflet écrivait dans l'introduction de sa thèse La filière bétail et viande bovine: « Le concept de filière n'aboutit pas, du moins à l'heure actuelle, à un corps théorique précis, à une référence suffisamment claire et assurée qui permettrait de charpenter tout discours sur toute filière » (21). En 1989, présentant le $\mathrm{X}^{\mathrm{e}}$ séminaire d'économie et de sociologie du CIRAD intitulé «Economie des filières en régions chaudes », Griffon regrettait aussi que ce concept ne soit pas cadré par une théorie et s'interrogeait sur le rôle que pouvaient jouer les thèses des économistes institutionnalistes (13).

Ce manque de cadre se retrouve dans les définitions qui peuvent être plus ou moins restrictives. Dans une conception assez large, « la filière représente l'ensemble des agents économiques, transformateurs ou non, des agents administratifs et politiques qui jalonnent directement ou indirectement l'itinéraire d'un produit du stade initial de la production (et des fournitures à la production) au stade final de la consommation (ou du moins de la transformation entrainant la perte d'identité du produit) ; elle est composée de toutes les interactions entre ces divers agents » (6). D'une façon plus restrictive, la filière peut être définie comme « une succession d'activités étroitement imbriquées les unes par rapport aux autres, liées verticalement à un même produit ou à des produits voisins et dont l'objectif principal, dans les filières agro-alimentaires, semble être de répondre aux besoins des consommateurs » (19).

\section{Les outils méthodologiques}

S'il n'existe pas de méthode standard, la consultation des différents auteurs attachés à cette méthodologie permet tout de même de décrire tout une palette d'outils.

\section{- La délimitation de la filière}

« Cette phase consiste à fournir une définition précise des produits retenus (matière première ou produits finis), à délimiter sur le plan vertical (hauteur), horizontal (largeur) et en volume (épaisseur) l'étendue de la filière et à préciser les espaces géographique et temporel sur lesquels la filière va être décrite » (18). C'est une étape stratégique qui doit permettre le « découpage » le plus pertinent possible pour répondre à la problématique de départ. 
Ainsi l'étude au Zimbabwe a été limitée aux produits viande de chèvre et cinquième quartier. La valorisation des peaux n'a pas été abordée. Le problème du lait ne se posait pas dans ce pays. Il s'agit en effet d'un exemple de filière caprine valorisant uniquement la viande, à l'inverse des pays du Maghreb et d'Afrique de l'Ouest où le lait de chèvre est aussi un produit.

La filière a été analysée du consommateur au producteur en tenant compte de tous les acteurs intermédiaires impliqués dans la production, la transformation et la distribution de chèvres ou de produits caprins. Ces acteurs pouvaient être des entreprises industrielles ou familiales, étatiques ou privées, intervenant dans le circuit formel ou informel. Le système d'amont n'a pas été l'objet d'étude. Celui-ci était quasi inexistant : les chèvres n'étaient absolument pas complémentées, laissées généralement en divagation, et les soins vétérinaires étaient considérés comme trop coûteux.

L'analyse filière a été menée à deux niveaux d'échelle, l'un provincial, l'autre national. Le premier centre d'intérêt était évidemment la province de Masvingo, cible du projet. Il était cependant important de replacer la filière provinciale dans le paysage national des productions caprines chaque fois que cela était possible afin de comprendre les interactions avec le reste du pays. Enfin le bilan dressé a été ponctuel et a correspondu à un instantané de la filière en 1994. Un bilan dynamique n'a pas pu être établi, il aurait demandé de prendre en compte la dernière grande sécheresse de 1991-1992 pour laquelle l'information aurait été difficile à obtenir et à vérifier.

\section{- La représentation}

La filière étant délimitée, il est nécessaire de la modéliser afin d'avoir un véritable outil de réflexion et d'analyse. On peut retenir quatre étapes : l'identification des flux et des opérations, l'identification des agents, l'analyse fonctionnelle, et enfin l'établissement du graphe de la filière (9)

\section{- La quantification physique des flux}

Fabre définit les flux comme « tous les transferts de biens, de services ou de fonds (argent, «droits économiques»de tous ordres) ». Il préconise ensuite l'utilisation d'une « matrice des flux croisant les agents entre eux et faisant apparaître la nature du produit qui les lie » (9).

\section{- L'analyse comptable}

Il s'agit d'un des éléments clés de l'étude de filière. Elle se base sur la notion de valeur ajoutée (VA) mesurant la création de richesses. Elle procède généralement en deux étapes : l'établissement des comptes d'agents, puis du compte consolidé de l'ensemble de la filière. Pour chaque type d'agent identifié par rapport à sa fonction principale, on cherche à établir un compte de production et un compte d'exploitation sur le modèle des comptes de branche. Le premier compte permet le calcul de la VA et retrace les opérations sur biens et services. Il peut être établi à partir du compte d'exploitation de l'agent au sens de la comptabilité d'entreprise. Le compte d'exploitation permet quant à lui de mettre en évidence la répartition de la VA.

Dans le cadre de la filière viande caprine au Zimbabwe, cette approche comptable sert de cadre de réflexion mais ne peut pas être appliquée à la lettre. Pour les producteurs, par exemple, évoluant dans un système très éloigné de l'économie de marché, les coûts de production sont très difficiles à estimer car aucun des facteurs de production n'est acheté. Il s'agirait en fait de coûts d'opportu- nité, qui sont quasi nuls du fait de l'absence d'utilisation alternative de la main d'œuvre et des parcours. L'analyse comptable des autres agents n'est pas plus aisée. Il est souvent impossible de réaliser les comptes analytiques nécessaires pour isoler la part des activités liée à la seule filière étudiée. La même chaîne d'abattage, par exemple, peut servir aussi bien aux bovins qu'aux caprins. Il est alors souvent impossible de calculer la VA et il faut se contenter d'une estimation de la marge brute réalisée avec le produit en question. Il n'est donc pas toujours aisé de dépasser le stade du compte d'exploitation de l'entreprise.

- La « mésoanalyse institutionnelle »

Constatant l'insuffisance d'une analyse comptable pour comprendre le fonctionnement d'une filière, Hugon a développé le concept de mésoanalyse institutionnelle qui prend en compte l'environnement géographique, politique et socio-économique des filières $(14,15)$.

En reprenant son analyse et en y intégrant l'approche développée à l'Institut national de recherche agronomique (INRA), par Lauret et Boutonnet, on peut définir six éléments primordiaux pour identifier les modes de régulation et caractériser ainsi différents soussystèmes productifs au sein d'une même filière :

\section{- Le niveau technologique}

Les sous-systèmes seront classés en artisanal ou industriel selon le degré de technicité (structure et capacité d'abattage, maîtrise de la chaîne du froid). Les formes artisanales, très nombreuses au Zimbabwe, ont donc des frais fixes moins importants mais une activité par opérateur plus réduite. Ces deux sous-systèmes entretiennent des relations complexes de concurrence et de complémentarité.

\section{- Les espaces de référence géographique}

Les différentes sous-filières n'ont pas le même champ d'action. On peut définir quatre niveaux : le local, c'est-à-dire généralement une activité limitée à l'échelle d'un district, le provincial, le national et l'international. Ces différentes échelles impliqueront des coûts, une organisation, des rapports entre agents très différents.

\section{- Les modes de coordination}

L'économie s'appuie sur deux piliers pour fonctionner : le marché, caractérisé par une coordination par le prix, et l'organisation entrấnant une intégration plus ou moins forte. Le premier de ces piliers apporte souplesse et capacité d'adaptation, le second, sécurité et rente. L'importance relative de ces deux éléments varie selon les différentes sous-filières existant au Zimbabwe, modifiant fortement le comportement des acteurs et donc le mode de formation des prix.

\section{- Le rôle de l'Etat}

En plus des deux modes de coordination qui viennent d'être cités, intervient l'Etat qui constitue aussi un élément clé de la caractérisation des sous-systèmes productifs. Ce dernier intervient, soit comme acteur économique, à différents niveaux d'intervention (entreprise nationalisée, paraétatique ou subventionnée), soit comme élément de contrôle et de codification. Dans le cas de l'abattage par exemple, les seuls opérateurs habilités à abattre des animaux pour en revendre la viande sont les abattoirs agréés par les services vétérinaires. Selon la place occupée par l'Etat, ou le degré de contrôle ou d'acceptation de la législation, le mode de fonctionnement d'un sous-système productif sera différent.

\section{- Les modes de concurrence}

Les différents modes de concurrence classiquement identifiés en fonction de l'offre et de la demande peuvent a priori exister et se- 
ront liés à des stratégies différentes. On note cependant que dans le cas des principaux vendeurs que sont les producteurs des zones communales, l'offre reste toujours dispersée. En revanche, les acheteurs peuvent parfois se trouver en situation de quasi-monopole pour des raisons techniques, devenant ainsi des price-makers.

\section{- Les logiques des acteurs}

La distinction des différents modes d'utilisation de la viande de chèvre fait déjà apparaître des logiques très différentes entre certains acteurs. La chèvre ne peut pas être vue comme une simple source de protéines. Ce produit n'a pas qu'une fonction alimentaire « biologique ». Les rôles " hédonique » et surtout « identitaire » ne doivent pas être négligés (5). Les abats restent la partie la plus appréciée par les consommateurs. Enfin, la chèvre représente un élément clé de la vie sociale : elle sert au paiement de la lobola (dot du futur marié), elle est de plus traditionnellement consommée à l'occasion de fêtes et fait l'objet d'une recherche particulièrement active à Noël par exemple. Mais, dans ces différents cas, la chèvre est recherchée vivante et, n'est en aucun cas déjà abattue et découpée.

\section{Les réalisations}

Les différents points méthodologiques qui viennent d'être présentés ont donc servi de base à la recherche d'information sur le terrain.

Trois enquêtes ont été réalisées afin d'approcher les principaux acteurs identifiés, d'estimer la part de la chèvre dans leur activité économique en se basant sur leur comptabilité, d'identifier les circuits d'approvisionnement et d'écoulement, de quantifier les flux d'animaux vivants ou morts, et enfin, de connaitre les objectifs et les souhaits de ces opérateurs pour le secteur caprin.

L'enquête auprès des producteurs n'a été menée que dans la province de Masvingo, zone ciblée par le projet (12). Cette province se divise en sept districts représentant $56566 \mathrm{~km}^{2}$. La population totale était estimée en 1992 à 1200000 habitants. L'enquête s'est limitée à quatre districts (Bikita, Chivi, Chiredzi et Mwenezi). Elle a été réalisée entre avril et juillet 1994, sur un échantillon de 4000 exploitations, par 12 enquêteurs organisés en deux équipes.

L'enquête auprès des abattoirs a couvert le territoire national et a concerné 32 abattoirs qui ne se sont pas tous avérés impliqués dans l'abattage de chèvres.

Enfin, l'enquête auprès des bouchers a été réalisée en deux phases. Au niveau national, elle s'est limitée à collecter des informations provenant d'une enquête auprès de bouchers, réalisée par le centre national de statistiques (CSO : Central statistical office). Au niveau de la province de Masvingo, une enquête faisant appel à un questionnaire spécifique, établi après l'étude des résultats du CSO, a été réalisée entre avril et juin 1995. Trente-huit bouchers possédant 45 boucheries au total ont été approchés.

Outre ces trois enquêtes, des entretiens informels ont permis d'approcher de nombreuses personnes impliquées dans la commercialisation de viande de chèvre. Leurs commentaires ont été reçus comme des avis d'experts connaissant particulièrement bien ce secteur d'activité. Ces entretiens ont aussi permis d'appréhender l'activité des supermarchés, de préciser la politique de l'entreprise paraétatique d'abattage et de stockage (CSC : Cold storage commission), de faire le bilan des politiques de subvention, d'obtenir des informations touchant au secteur informel, en particulier le rôle des intermédiaires, et de recueillir des avis sur les possibilités de développement de cette filière caprine.

\section{RESU LTATS}

La réalité économique d'un système de production ne peut jamais être appréhendée dans son ensemble et dépend, en quelque sorte, de l'angle d'observation. Ainsi, toute représentation de la réalité est partielle, privilégiant certains éléments et certaines relations.

L'étude de la filière viande de chèvre au Zimbabwe a permis de réaliser deux modèles explicatifs, axés sur des problématiques différentes mais complémentaires.

\section{Un premier modèle basé sur la dichotomie formel-informel}

Le manque de données statistiques fiables et exhaustives a beaucoup influencé le choix du premier découpage basé sur la dichotomie formel-informel (20). Une des caractéristiques de la filière viande caprine au Zimbabwe est, en effet, la présence d'un secteur informel très développé.

Deux aspects sont pris en compte pour définir la notion d'informel (1) :

- le premier est l'enregistrement ou non dans les statistiques nationales : le secteur informel se définit alors comme l'ensemble des opérateurs dont l'activité échappe à tout recensement, ou encore, comme le secteur caché ;

- le second aspect touche à la réglementation qui régit le secteur d'activité : le secteur informel est composé des agents qui opèrent de façon illégale. Dans le cas de l'abattage, par exemple, d'un point de vue légal, les seuls opérateurs habilités à abattre des animaux pour en revendre la viande sont les abattoirs agréés par les services vétérinaires.

En tenant compte de ces deux critères, on se rend compte que l'essentiel de l'activité caprine est réalisé dans le secteur informel. Les producteurs sont les plus gros consommateurs de viande de chèvre : plus de la moitié des animaux exploités sont autoconsommés et n'entrent donc dans aucun circuit de commercialisation. Cette autoconsommation des chèvres entre évidemment dans le secteur informel.

Mais les circuits de commercialisation sont aussi dominés par le secteur informel. N'entrent dans les circuits formels que les animaux abattus par la CSC et les abattoirs agréés ainsi que les animaux sur pied exportés de façon officielle $(7,8)$. Or ces circuits ne touchaient que 45000 caprins en 1994. Des circuits de commercialisation courts de type producteur-consommateur ou de type producteur-boucher-consommateur gèrent le reste des animaux mis sur le marché, soit 445000 têtes en 1994.

En se basant sur les résultats des enquêtes et en prenant donc comme hypothèse un cheptel national de 3,5 millions de têtes, un taux d'exploitation de 32 p. 100 et une autoconsommation de 630000 animaux pour 1994, les circuits informels de commercialisation représenteraient 96 p. 100 de la consommation de la viande de chèvre.

Ce premier modèle (figure 2) permet de décrire, sur les deux types de marché, formel et informel, les relations entre les opérateurs, depuis le producteur jusqu'au consommateur, tout en tenant compte des flux d'animaux vivants, de carcasses et de viande découpée (3).

Mais le positionnement des différents opérateurs par rapport au secteur formel entraîne certaines ambiguïtés : un boucher, par exemple, peut très bien s'approvisionner en partie auprès d'un 


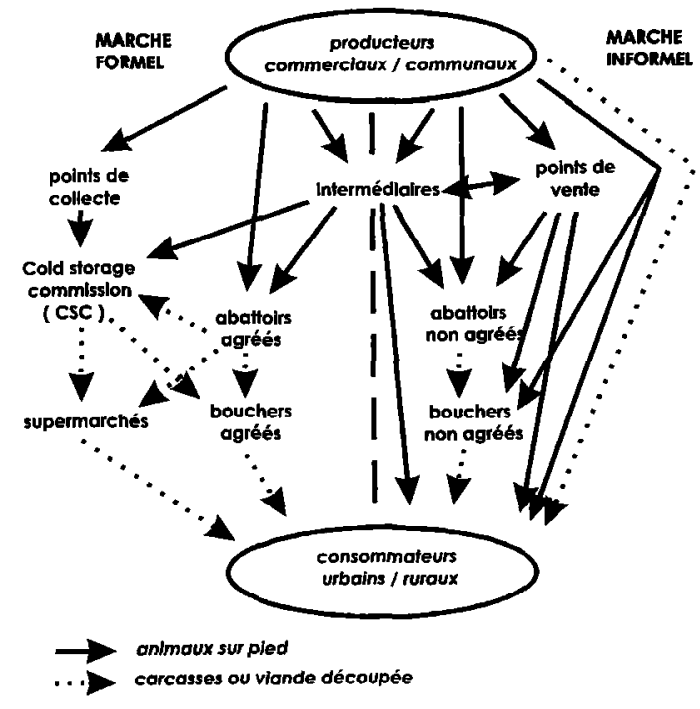

Figure 2 : la filière viande caprine au Zimbabwe, une première reprësentation.

abattoir agréé, et donc travailler dans le secteur formel, tout en abattant lui-même une partie des animaux sans autorisation, et donc en étant considéré dans le secteur informel.

Ce découpage ne permet pas non plus de faire apparaître les différentes stratégies développées par les acteurs. Le secteur informel englobe en effet des personnes ayant des logiques opposées : le boucher recherche un approvisionnement régulier à un prix acceptable pour pouvoir vendre de la viande au détail en faisant une marge suffisante ; le particulier, en revanche, peut vouloir rechercher un animal sur pied pour une occasion sociale se déroulant souvent à une date précise et est prêt à y mettre le prix qu'il faut pour l'obtenir.

Insuffisant pour rendre compte des stratégies existantes au sein de la filière viande de chèvre, le découpage formel-informel doit donc être dépassé pour aborder cette filière avec d'autres critères discriminants.

\section{Vers un autre découpage de la filière viande de chèvre au Zimbabwe}

A partir des critères discriminants choisis en s'appuyant sur la mésoanalyse institutionnelle, cinq sous-filières peuvent être identifiées. Il est à noter que seuls les trois premiers types de circuits de commercialisation identifiés par Boutonnet et Simier sont retrouvés dans leur ouvrage Les viandes (4). Les circuits faisant appel à deux grossistes, essentiellement observés pour les échanges lointains, n'existent pas au Zimbabwe dans le cas de la filière viande de chèvre.

\section{(a) La vente directe}

Les ventes directes représentent actuellement la majorité des flux du secteur caprin. On peut se baser sur l'enquête production réalisée dans la province de Masvingo pour estimer ces flux. Une vente de plus de 3000 chèvres a été enregistrée pour la période d'avril 1993 à avril 1994. Dans 95 p. 100 des cas les acheteurs étaient des particuliers, dont 37 p. 100 des fermiers, et 24 p. 100 des instituteurs. Les fermiers peuvent cependant acheter à des fins de reproduction et non pas seulement d'abattage.

Le prix payé est un prix à la tête sans que personne ne sache le poids de l'animal. Le degré de technicité, le long de cette sous-filière, est donc quasi nul. En outre, aucune amélioration de productivité n'est envisageable dans un tel système, un gain de poids discret ne pouvant pas être valorisé.

L'acheteur peut être motivé par deux raisons principales : il peut vouloir, s'il est lui-même éleveur, acquérir un animal pour la reproduction mais, dans la majorité des cas, il en a besoin pour une occasion sociale particulière ou pour l'une des grandes fêtes zimbabwéennes, et en particulier pour Noël. Pour toutes ces occasions, l'animal est généralentent abattu à domicile ; les abats, très appréciés, sont coupés en morceaux et frits ; la viande est préparée avec une sauce à base de tomates et d'oignons et accompagne la traditionnelle sadza (farine de maïs).

D'après l'enquête production, dans plus de 98 p. 100 des cas, ces particuliers, quelles que soient les raisons de leur achat, habitent dans le même district que le vendeur. Il s'agit d'un circuit local.

\section{(b) La sous-filière faisant intervenir un boucher-abatteur}

La situation de ces bouchers-abatteurs par rapport à la loi zimbabwéenne et au contrôle de l'Etat est très variable. Certains abattent illégalement et ne se font pas recenser pour éviter de payer leur licence. D'autres abattent illégalement mais sont recensés comme distributeurs. Enfin, certains bouchers avec licence obtiennent une dérogation des services vétérinaires pour abattre. En fait; le système législatif en vigueur semble inadapté à la réalité du terrain et entraîne souvent une absence de contrôle et dans le meilleur des cas la mise en place de dérogations avec, comme condition, le respect de la chaîne du froid.

Si l'on se référait à la dichotomie formel-informel, une grande partie de ces bouchers seraient classés dans le secteur informel. Ici, ils sont regroupés dans une même sous-filière présentant certaines caractéristiques particulières.

Une grande partie des bouchers paient aussi les animaux à la tête. Certains d'entre eux ont tout de même une idée du prix payé par kilogramme vif sachant à combien le kilogramme de carcasse leur est revenu. Une minorité de bouchers pratiquent la pesée et le paiement par kilogramme vif. Ils se situent généralement dans les zones commerciales.

L'abattage est réalisé par le boucher lui-même. La structure d'abattage est souvent des plus sommaires et l'éviscération et la découpe se font souvent à même le sol. Lorsque le volume de carcasses est plus important, on peut trouver un local équipé de plusieurs rangées de crochets permettant de suspendre les animaux une fois abattus. L'éviscération et le dépeçage se font alors de façon plus rapide et plus propre. Il s'agit souvent de boucheries ou chaînes de boucheries en zone périurbaine ou commerciale. La maîtrise du froid est très variable également. Lorsque l'activité d'abattage est importante, ce qui est souvent associé à une dérogation des services vétérinaires, les bouchers disposent tous de chambres froides. En revanche, dans des boucheries à faible activité générale, situées dans des lieux reculés des zones communales, il arrive souvent qu'il n'y ait aucun moyen de réfrigération.

D'une façon générale, les boucheries, grandes consommatrices de main d'œuvre bon marché, fonctionnent encore sur le mode artisanal et leurs coûts d'abattage et de distribution restent faibles. 
Cette activité se déroule toujours à un niveau local, aussi bien pour l'approvisionnement que pour la collecte. Même si les auteurs ont rencontré un propriétaire de plusieurs boucheries à Chiredzi qui était prêt à sortir de son district pour aller collecter des chèvres, sa clientèle reste locale. Dans tous les cas, ces bouchers abattent pour vendre au détail eux-mêmes. Aucun n'a une activité de grossiste.

La plupart de ces bouchers s'occupent aussi de la collecte. Dans les zones communales, celle-ci n'est pas réellement un problème, les boucheries étant installées dans les zones de production et leur demande restant faible. Dans les zones périurbaines ou commerciales, elle devient plus difficile à gérer : les bouchers ont peu de prises sur la production qui est très éparpillée ; la recherche laborieuse des animaux disponibles à la vente, entraînant des coûts de déplacement élevés, les décourage souvent. Leurs moyens de transport sont variables : parfois inexistants, il s'agit généralement de véhicules de type pick-up ; certains bouchers de zones commerciales disposent aussi de camions servant généralement pour les bovins.

\section{(c) La sous-filière faisant intervenir un abattoir agréé}

L'abattage est ici assuré par des entreprises spécialisées et dont les locaux répondent aux normes zimbabwéennes. Le niveau technique est donc plus élevé. L'abattage est réalisé à la chaîne, les carcasses sont conservées dans des chambres froides et les livraisons se font avec des camions frigorifiques. Ces abattoirs disposent aussi généralement de camions pour le transport des bovins vivants qu'ils peuvent occasionnellement utiliser pour des caprins mais le nombre minimum exigé est alors élevé.

Leur zone d'action, aussi bien au niveau de la collecte que de la livraison, est généralement à l'échelle de la province, sauf dans un cas rencontré à Bulawayo où les livraisons sont réalisées à l'échelle nationale.

Lorsque la collecte est assurée par l'abattoir, celui-ci utilise des points de rassemblement communaux servant généralement pour des bovins. Mais, ces points de rassemblement étant rares, il se trouve souvent en concurrence avec d'autres grossistes et peut faire le déplacement pour rien. Ce manque de maîtrise de l'approvisionnement, pour lequel beaucoup d'entre eux ne veulent pas investir car la chèvre représente une faible part de leur activité, les pousse à opter pour une politique opportuniste et attentiste : certains d'entre eux ne travaillent donc qu'avec les livraisons occasionnelles de producteurs; d'autres se reposent sur des intermédiaires mais leur activité ne semble pas très importante.

Le prix payé au producteur est un prix par kilogramme vif qui subit des variations, au cours de l'année, en fonction des disponibilités.

La clientèle de ces abattoirs est constituée essentiellement de bouchers, occasionnellement de supermarchés. Ces derniers ne sont pas très appréciés à cause de leur mode de paiement décalé. D'une façon générale, l'irrégularité des livraisons de carcasses de chèvres ne permet pas aux abattoirs de fidéliser leur clientèle, tout du moins pour ce produit.

\section{(d) La sous-filière faisant intervenir la CSC}

La CSC est le numéro un des abatteurs au Zimbabwe. Ses abattoirs sont tous aux normes CEE ce qui lui permet de gérer, entre autres, les exportations de viande bovine. Son réseau de collecte et de livraison couvre tout le territoire. L'abattoir des petits ruminants, construit à l'image de ceux réservés aux bovins, est surdimensionné et n'a jamais tourné à plein régime.
L'histoire de cet office est en fait liée à la culture de l'élevage bovin. Cette production, très présente sous l'ancien régime colonial, est restée un des fleurons de l'agriculture zimbabwéenne et a donné à la CSC ses lettres de noblesse. A partir de 1986, l'Etat lui a demandé de s'occuper aussi des productions caprines associées aux zones communales, mais son intervention apparaît plus civique que commerciale.

Elle ne se donne d'ailleurs pas réellement les moyens de cette politique. Ses camions sont inadaptés au transport des caprins : trop grands, ils nécessitent des collectes de 300 chèvres pour rentabiliser les déplacements et, de plus, le taux de mortalité au cours du transport est élevé. Sa politique des prix, évoquée dans l'analyse comptable, n'est pas non plus très dynamisante.

Malgré cela, les producteurs restent relativement fidèles à la CSC car ils peuvent profiter de son réseau de points de collecte tout au long de l'année. Même si le transport est organisé pour des bovins, la CSC ne refuse jamais de prendre en plus quelques chèvres pour dépanner les éleveurs. Ainsi les fermiers ont toujours un marché pour leur animaux, à un prix par kilogramme vif garanti, même dans des périodes où la demande est plus faible.

\section{(e) La sous-filière faisant intervenir une structure de type coopérative}

Cette sous-filière ne sera pas réellement étudiée. Elle est en effet plutôt anecdotique car elle ne touche qu'un groupe de fermiers commerciaux propriétaires de leur propre abattoir sur Harare. Elle est tout de même intéressante parce qu'elle montre un exemple d'intégration verticale, l'abattoir fonctionnant comme une coopérative dont sont membres les différents producteurs.

Le circuit d'exportation d'animaux vivants n'a pas été traité non plus puisqu'il ne concerne qu'un opérateur qui le fait de façon occasionnelle (6 000 caprins en 1994).

La figure 3 permet de schématiser ces différents sous-systèmes, le tableau I fait le point par rapport aux critères discriminants retenus.

Par souci de simplification, les interactions entre les sous-filières n'ont pas été non plus représentées mais il est évident qu'un même boucher peut s'approvisionner de différentes façons.

Cette formalisation ne permet cependant pas de mettre en avant certains éléments décrits dans ces critères discriminants. Reprenant un mode de présentation utilisé par Lauret et Boutonnet, on peut chercher à positionner les sous-systèmes productifs par rapport à deux axes tenant compte de ces critères (figure 4). On obtient alors un mode de représentation complémentaire à celui exposé précédemment et permettant de mieux comprendre le fonctionnement des différentes sous-filières.

Ainsi le premier axe s'intéresse aux modes de coordination. Les sous-filières (e) (coopérative) et (d) (CSC) représentent les deux sous-systèmes productifs les plus organisés. Les producteurs sont fortement liés aux structures d'abattage, pour des raisons de sécurité et de facilité dans le cas de la CSC, d'intérêt dans le cas de la coopérative. Le prix d'achat est un prix fixé. Les trois autres sousfilières sont en revanche plus soumises à la loi du marché : le prix suit la loi de l'offre et de la demande.

Le deuxième axe s'intéresse au rôle de l'Etat. Il est clair que la CSC est la structure la plus liée à l'Etat. On peut remarquer l'absence d'une participation de l'Etat par le moyen des subventions. Son rôle reste législatif pour les autres structures. On doit d'ailleurs s'inquiéter de la position de la sous-filière (b) qui, du fait d'une législation inadaptée, se retrouve souvent dans des situations illégales, rendant délicate la mobilisation de ses agents pour un projet de développement. 


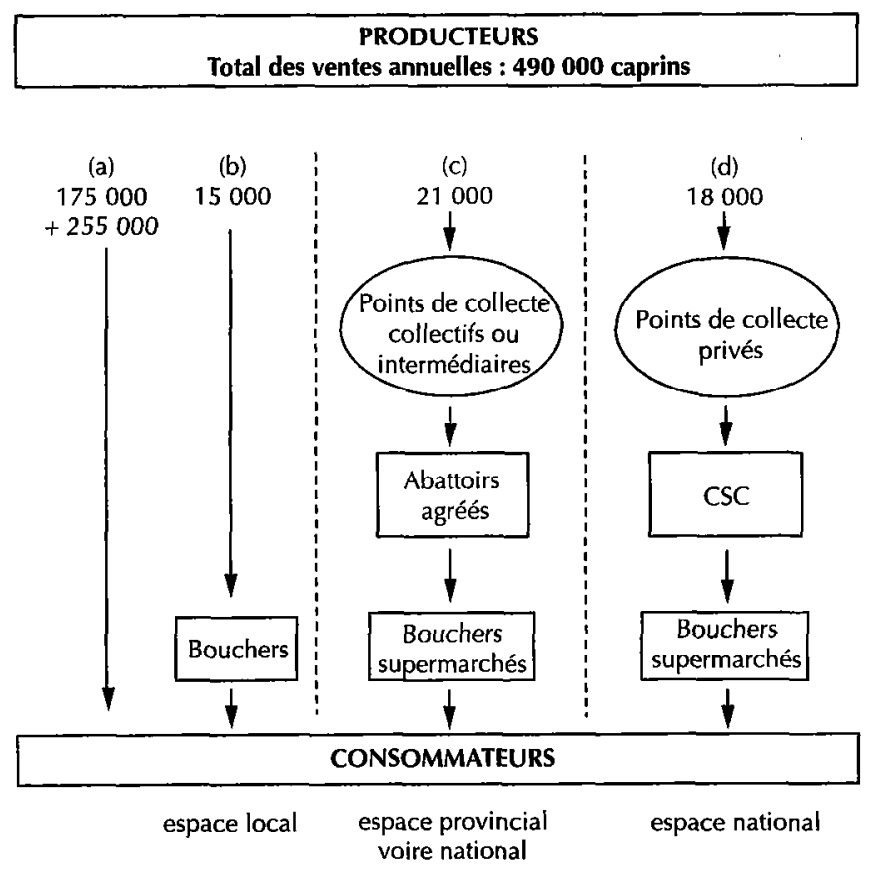

Les estimations numériques ont été réalisées à partir des enquêtes et des hypothèses retenues dans l'article. L'effectif national est donc de 3,5 millions et le taux d'exploitation, de 32 p. 100. 630000 animaux ont été autoconsommés, 490000 têtes ont donc été commercialisées. Le taux de renouvellement ayant été établi, par enquête, à 5 p. 100, le chiffre de 175000 affiché dans la sous-filière (a) correspond aux achats d'animaux reproducteurs. Le nombre total de chèvres consommées au Zimbabwe en 1994 s'élevait donc à 939000 animaux, soit une consommation annuelle par habitant de $1,1 \mathrm{~kg}$ en prenant comme poids carcasse moyen, $13 \mathrm{~kg}$. Enfin, la figure 3 ne fait pas apparaître les 6000 têtes exportées cette même année.

Figure 3 : les différents sous-systèmes productifs de la filière viande de chèvre au Zimbabwe.

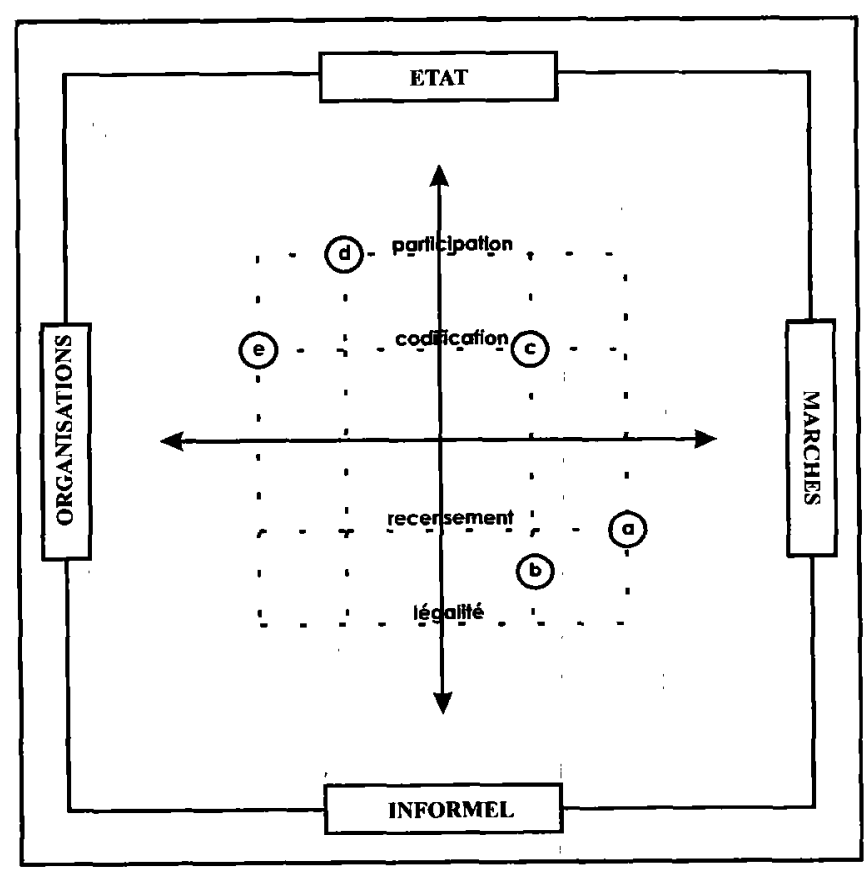

(a) la vente directe

(b) la sous-filière faisant intervenir un boucher-abatteur

(c) la sous-filière faisant intervenir un abattoir agréê

(d) la sous-filière faisant intervenir la CSC

(e) la sous-filière faisant intervenir une structure de type coopérative

Figure 4 : position des sous-filières sur les axes Etat-informel et marchés-organisations.

\section{Tableau I}

Typologie des différentes sous-filières en fonction des critères discriminants de la mésoanalyse institutionnelle

(a)

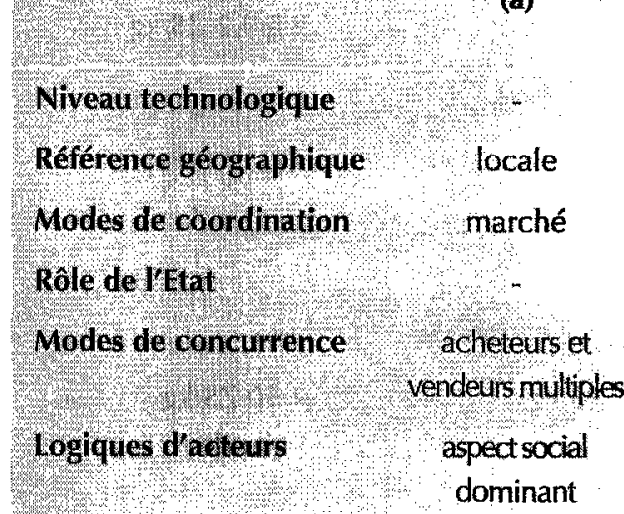

(b)

+
locale
marché
+
cheteurs et
deurs multiples
dominant

(c)

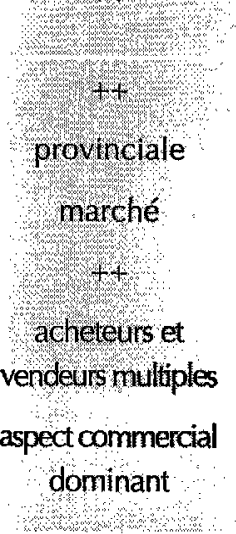

(d)

(e)

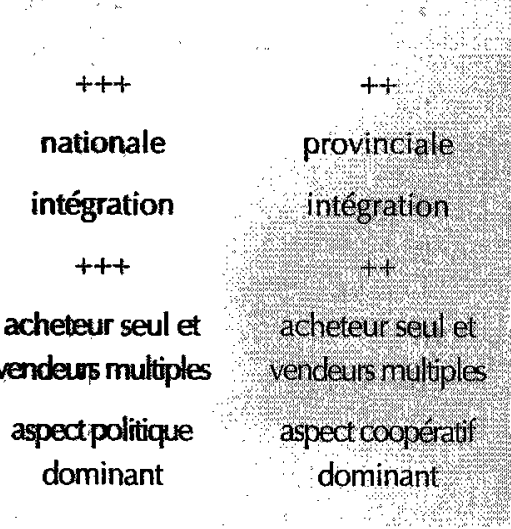

(a) la vente directe

(b) la sous-filière faisant intervenir un boucher-abatteur

(c) la sous-filière faisant intervenir un abattoir agréé

(d) la sous-filière faisant intervenir la CSC

(e) la sous-filière faisant intervenir une structure de type coopérative 


\section{COMMENTAIRES ET PRO SPECTIVES}

Ayant choisi de ne pas revenir sur le premier modèle qui permet de visualiser les différents acteurs et les flux, les auteurs se sont intéressés, en revanche, au second modèle, basé sur l'identification de sous-systèmes productifs présentant des modes de fonctionnement différents.

L'établissement des comptes par rapport aux différentes sous-filières identifiées représente un élément supplémentaire pour comprendre les logiques de fonctionnement et, surtout, vérifier que le discours est cohérent par rapport à la réalité comptable. L'étude de la décomposition des prix, et donc des marges réalisées, a été effectuée pour les trois principales sous-filières (autre que la vente directe) faisant intervenir comme abatteur la CSC, un abattoir agréé ou un boucher. Ces différents circuits de commercialisation sont présents dans la province de Masvingo qui était ciblée par le projet. Les résultats figurent dans le tableau II.

La première constatation qui pourrait être faite est la faible marge de manœuvre des distributeurs. Leur profit reste modeste par rapport à celui des abattoirs. Leur demande en carcasses, supérieure à l'offre du marché, ne leur permet pas de discuter le prix de gros et la hausse du prix de détail est limitée par les prix des autres viandes. Même si la viande de chèvre bénéficie d'une aura «identitaire » indéniable, ce produit reste d'une qualité moyenne. C'est une viande dure qui n'est préparée qu'en sauce. Elle s'adresse de plus à la population africaine du pays dont la grande majorité se caractérise par de faibles revenus.
Pour rester attractif, le prix doit donc être inférieur à celui que le consommateur doit payer pour les autres viandes, soit $19 \mathrm{Z \$}$ par kg pour le bœuf, $20 \mathrm{Z} \$$ par kg pour le mouton, 17,2 Z \$ par kg pour le porc et $18,4 \mathrm{Z} \$$ par $\mathrm{kg}$ pour le poulet.

Ces raisons expliquent le choix de certains bouchers d'abattre euxmêmes, d'autant plus qu'ils bénéficient ainsi de la valorisation du $5^{\mathrm{e}}$ quartier et de la peau.

Le prix de détail en boucherie diffère d'une localité à l'autre et reflète la rareté relative de la viande de chèvre. Dans les zones communales (exemple de Chivi), il n'y a pas de forte demande de viande de chèvre dans les boucheries car les consommateurs peuvent et préfèrent s'en procurer par eux-mêmes. Le prix de la viande de chèvre est donc plus bas qu'ailleurs et ne permet pas aux bouchers de s'approvisionner auprès des abattoirs. Si l'abattage est effectué par le boucher lui-même, il peut alors prendre une marge confortable (de 5,3 à 6,6 Z\$ par kg, soit 36 à 45 p. 100 du prix de détail).

Dans la zone de Chiredzi, les auteurs ont noté que les bouchers n'arrivent pas souvent à s'approvisionner à la hauteur de leurs besoins, ce qui laisse penser que la demande en viande de chèvre est supérieure à l'offre. Par conséquent, le prix de détail y est supérieur de $1 \mathrm{Z} \$$ par kg à celui observé à Chivi. Les bouchers abattant eux-mêmes peuvent alors proposer un prix plus attractif pour les producteurs tout en conservant une marge correcte (de 4,25 à 4,5 Z \$ par kg, soit 27 à 29 p. 100 du prix de détail).

Si la marge des distributeurs faisant appel à un grossiste est faible, celle de ce dernier est en revanche plus large.

\section{Tableau II}

Décomposition du prix selon les différentes sous-filières

\begin{tabular}{|c|c|c|c|c|}
\hline & \multirow[t]{2}{*}{ CSC } & \multirow[t]{2}{*}{ Abattoir agréé } & \multicolumn{2}{|c|}{ Boucher abatteur } \\
\hline & & & Chiredzi & Chivi \\
\hline \multirow{2}{*}{$\begin{array}{l}\text { Prix à la porte } \\
\text { de la ferme (1) }\end{array}$} & $3,00 \mathrm{Z \$} / \mathrm{kg}$ vif & $*$ & $3,8 \mathrm{Z \$} / \mathrm{kg}$ vif & 3,0 à $3,5 \mathrm{Z \$} / \mathrm{kg}$ vif \\
\hline & 7,90 Z\$/kg carc. & & 10,0 Z\$/kg carc. & 7,9 à $9,2 \mathrm{Z} \$ / \mathrm{kg}$ carc. \\
\hline Coûts de transport & 1,05 Z\$/kg carc. & $*$ & $1,0 \mathrm{Z} \$ / \mathrm{kg}$ carc. (4) & $0,0 \mathrm{Z} \$ / \mathrm{kg}$ carc. \\
\hline Prix rendu abattoir & 8,95 Z\$/kg carc. & $10,0 \mathrm{Z} \$ / \mathrm{kg}$ carc. & 11,0 Z\$/kg carc. & 7,9 à $9,2 \mathrm{Z} \$ / \mathrm{kg}$ carc. \\
\hline Coûts d'abattage (2) & 1,10 Z\$/kg carc. & $0,6 \mathrm{Z} \$ / \mathrm{kg}$ carc. & $?$ & $?$ \\
\hline Coûts de livraison & 0,35 Z\$/kg carc. & $0,0 \mathrm{Z} \$ / \mathrm{kg}$ carc. & $*$ & $*$ \\
\hline Profit & $0,60 \mathrm{Z} \$ / \mathrm{kg}$ carc. & 0,4 Z\$/kg carc. & $?$ & $?$ \\
\hline Prix de gros & $11,00 \mathrm{Z} \$ / \mathrm{kg}$ carc. & $11,00 \mathrm{Z} \$ / \mathrm{kg}$ carc. & $*$ & $*$ \\
\hline $\begin{array}{l}\text { Coûts de } \\
\text { distribution (3) }\end{array}$ & 4,25 Z\$/kg carc. & 4,25 Z\$/kg carc. & $?$ & $?$ \\
\hline Profit & 0,15 Z\$/kg carc. & $0,15 \mathrm{Z \$} / \mathrm{kg}$ carc. & $?$ & $?$ \\
\hline Prix de détail & $\begin{array}{l}15,40 \mathrm{Z} \$ / \mathrm{kg} \\
\text { viande }\end{array}$ & $\begin{array}{c}15,40 \mathrm{Z} \$ / \mathrm{kg} \\
\text { viande }\end{array}$ & $\begin{array}{c}15,5 \mathrm{Z} \$ / \mathrm{kg} \\
\text { viande }\end{array}$ & $\begin{array}{c}\text { 14,50 Z\$/kg } \\
\text { viande }\end{array}$ \\
\hline
\end{tabular}

(1) calculé avec rendement carcasse de 38 p. 100

(2) comprend : salaires, coût financier, consommations intermédiaires

(3) comprend : licence, salaires, électricité, loyer, réparations et dépréciation, coûts administratifs, emballages

(4) transport de petite capacité ( $20 \mathrm{cts} / \mathrm{km} / \mathrm{chèvre)} \mathrm{;} \mathrm{distance} \mathrm{moyenne} \mathrm{de} 65 \mathrm{kms}$

Le profit, établi pour les différents abatteurs, ne prend pas en compte la valorisation du cinquième quartier (abats, tête et pieds) et de la peau qui représentent $30 \mathrm{Z} \$$ par chèvre, soit un profit net supplémentaire de 2 Z\$ par kg de carcasse. Les prix sont exprimés en dollars zimbabwéens courants. En 1994, 1 dollar zimbabwéen valait 0,6 franc français 
Malgré des coûts de transport (approvisionnement et livraison) élevés absorbant 45 p. 100 de sa marge, la CSC est l'abattoir qui réalise la plus grosse marge par kilogramme de carcasse. Elle jouit, en fait, d'une situation de rente ayant fidélisé en quelque sorte certains producteurs, du fait de son image de marque et de son réseau de points de collecte. Ce constat repose la question de la stratégie de la CSC qui ne semble pas vouloir réaliser, dans les actes, son discours politique d'amélioration du revenu des petits producteurs en leur permettant de valoriser leurs produits caprins.

Dans le cas des abattoirs privés, les coûts sont évidemment plus faibles que ceux d'une grosse structure, comme la CSC, travaillant au niveau national. Il faut aussi rappeler que la CSC a un abattoir spécial pour les petits ruminants qui ne fonctionne qu'à 17 p. 100 de sa capacité d'abattage. Les frais fixes ramenés au kilogramme de carcasse sont donc très importants. Inversement, l'abatteur privé possède généralement une petite structure qui sert à la fois pour les bovins et les caprins de sorte que les frais fixes sont mieux couverts. Il peut donc se permettre d'avoir un prix au kilogramme de carcasse rendue abattoir plus élevé que celui de l'office paraétatique. Les auteurs ne disposaient pas dans cette étude de prix payé à la ferme. En considérant un transport de grande capacité et une distance moyenne de collecte de $100 \mathrm{~km}$, ce prix serait alors de 3,6 Z \$ par kg vif et donc nettement plus élevé que celui proposé par la CSC. Mais les différences de coût n'expliquent pas tout, la marge réalisée par l'abattoir est aussi plus faible, montrant une option stratégique différente. Ne disposant pas de réseau de collecte, ces abattoirs doivent donc être plus attractifs que la CSC s'ils s'intéressent à ce produit.

Les différentes sous-filières montrent ainsi des possibilités de croissance assez variables. La CSC, disposant pourtant d'infrastructures imposantes, ne mène pas de politique de croissance pour son activité chèvre. Son action semble surtout politique, dictée par l'Etat. Les interlocuteurs privilégiés identifiés au cours des enquêtes (abattoirs agréés, bouchers périurbains) appartiennent en revanche à des sous-systèmes productifs montrant plus d'intérêt pour le produit chèvre, dans leur discours et dans leurs actes.

Mais cette activité reste secondaire chez ces opérateurs. La viande bovine reste leur fonds de commerce. L'aspect marginal de la viande de chèvre ne justifie pas, malgré leur intérêt, l'investissement nécessaire pour organiser des collectes.

Dans le cadre d'un projet d'amélioration des circuits de commercialisation, l'organisation de la collecte des produits devrait donc s'appuyer sur ces opérateurs, demandeurs de circuits d'approvisionnement, et non pas sur la structure paraétatique de la CSC qui ne voit pas, en la chèvre, un produit à développer.

\section{- CONCLUSION}

Parti d'un état des connaissances sur la production caprine très incomplet, la mise en place d'une analyse de filière a permis aux auteurs de combler un grand nombre de lacunes concernant la connaissance du produit viande de chèvre et de son utilisation.

Guidé par un souci de pragmatisme et la constatation de l'inadéquation entre l'offre et la demande pour ce produit, ce choix méthodologique a abouti à plusieurs modélisations complémentaires de l'activité caprine.

La disproportion des effectifs concernés par les secteurs formel et informel a été quantifiée. Cette disproportion se retrouve dans le deuxième modèle, basé sur les critères discriminants de la mésoanalyse institutionnelle. Cette dernière approche, permettant d'analyser les contraintes de chaque sous-système productif ainsi que les stratégies associées, laisse entrevoir des possibilités de développement et permet donc d'apporter les réponses aux questions d'approvisionnement et de développement posées en introduction. Le circuit d'autoconsommation est saturé. Les circuits courts traditionnels n'ont pas besoin de l'appui du projet. En revanche, dans les circuits campagnes-villes, si la CSC apparaît inefficace à développer la filière caprine, certains autres acteurs tels que les bouchers périurbains et les abatteurs privés, par leurs pratiques (pesée des animaux) et leurs stratégies, apparaissent comme des interlocuteurs privilégiés. Ils devraient permettre de mener à bien un projet de renforcement des circuits de commercialisation de la viande de chèvre, dans une optique d'amélioration de l'approvisionnement des villes mais aussi d'intégration des petits producteurs dans l'économie de marché, afin d'entrainer, à long terme, un développement réel de la filière et non pas un simple transfert d'utilisation de la production. Plus concrètement, le renforcement des circuits dans lesquels se situent ces opérateurs devrait permettre, par une augmentation des prix payés aux producteurs et une plus grande facilité de vente-collecte, un accroissement de la production commercialisée. Cet accroissement est possible sans diminuer l'autoconsommation par une augmentation du cheptel et/ou une intensification de la production que les services techniques savent mettre en place, mais sans trouver jusque là de répondant faute de circuits.

La mise en place de points de collecte (10), dans le cadre du projet d'aide aux productions caprines dans la province de Masvingo, a d'ailleurs permis de vérifier la cohérence de l'analyse présentée : la majorité des acheteurs qui ont répondu à ce projet étaient des bouchers périurbains, un abattoir agréé s'est joint à cette action, les prix moyens pratiqués par jour de marché de collecte ont oscillé entre 3,33 et 4,55 Z\$ par kg vif rendant ces marchés attractifs pour les vendeurs.

\section{BIBLIO GRAPHIE}

1. ADAIR P., 1995. Economie informelle. Cah.GRATICE, 9 : 3-11.

2. BALLASTER F. et coll. (trentaine d'auteurs), 1994. Goat production in communal lands. Harare, Zimbabwe, CIRAD-EMVT Zimbabwe/Agritex, 173 p. (Manuel de formation)

3. BERIN STAIN-BAILLY C., 1992. La production de viande caprine en Afrique. Etude de cas : le Zimbabwe. Capricorne, 5 (4) : 9-14.

4. BOUTONNET JP., SIMIER JP., 1995. Les viandes. Paris, France, Economica, $110 \mathrm{p}$.

5. BRICAS N., 1995. Séminaire filières et marchés. M émoire DEA Economie du développement agricole, agro-alimentaire et rural, ENSA, Université de M ontpellier I, M ontpellier, France.

6. CHALMIN P., MOUTON P., 1982. L'analyse de filière appliquée aux marchés internationaux des produits agricoles. Paris, France, Economica.

7. CSO , 1994. Q uarterly digest of statistics. Zimbabwe. Harare, Zimbabwe, CSO, $60 \mathrm{p}$.

8. CSO, 1992. Statistical yearbook. Zimbabwe. Harare, Zimbabwe, CSO, $266 \mathrm{p}$.

9. FABRE P., 1994. Note de méthodologie générale sur l'analyse de filière : utilisation de l'analyse de filière pour l'analyse économique des politiques. Document de formation pour la planification agricole. Rome, Italie, FAO, $105 \mathrm{p}$.

10. GAUTHIER J., 1996. La filière viande de chèvre au Zimbabwe. Capricorne, 9 (1) : 12-16.

11. GAUTHIER J., 1996. Renforcement des circuits de commercialisation de la viande de chèvre dans la province de Masvingo, Zimbabwe : utilisation du concept de filière comme outil d'analyse. Mémoire DEA, Economie du développement agricole, agroalimentaire et rural, ENSA, Université de Montpellier I, Montpellier, France, $97 \mathrm{p}$. 
12. GAUTHIER 」, PRADIER A., SHUMBA C., 1995. Main results of the survey on goat marketing in Masvingo Province. Masvingo, Zimbabwe, CIRAD-EM VT/Agritex, 118 p. (Rapport interne)

13. GRIFFON M., 1989. Présentation du séminaire. In : Economie des filières en régions chaudes, $X$ Séminaire d'économie et de sociologie, Montpellier, France, 11-15 septembre 1989, p. 1-3.

14. HUGON P., 1989. Filières agricoles et programmes d'ajustement structurel. In : Economie des filières en régions chaudes, $X^{e}$ Séminaire d'économie et de sociologie, Montpellier, France, 11-15 septembre 1989, p. 7-11.

15. HUGON P., 1992. La mésoéconomie institutionnelle et l'agriculture africaine : le cas de la filière coton. In : XIIIe Séminaire d'économie et de sociologie, Montpellier, France, 7-9 septembre 1992.

16. LAURET F., 1983. Sur les études de filières agro-alimentaires. Econ. SOC., $5: 721-740$.

RET F., PEREZ R., 1992. Mésoanalyse et économie agroalimentaire. Econ. Soc., 6 : 99-118.

\section{Summary}

G authier J., Pradier A., Lecroisey F., Shumba C. Study of the goat meat commodity subsector in Zimbabwe

This study presents the goat sector in Zimbabwe with special emphasis on Masvingo Province and uses the production/marketing channel as an analysis tool. Following a brief explanation for this choice and a description of the methodology tools two channel models are proposed. The second more powerful model is discussed in relation with costs/returns. In conclusion, such an analysis reveals distinct players, who will help implement development activities aiming at strengthening goat meat marketing channels and increasing smallholders' income.

Key words: Goat meat - Marketing channels - Agricultural economics - Model - Zimbabwe.
18. MONTIGAUD J.C., 1989. Les filières fruits et légumes et la grande distribution : méthodes d'analyses et résultats. In : Economie des filières en régions chaudes, $X$ e Séminaire d'économie et de sociologie, Montpellier, France, 11-15 septembre 1989, p. 37-50.

19. MONTIGAUD J.C., 1989. L'analyse des filières agro-alimentaires: méthodes et premiers résultats. Econ. Soc., 6 : 59-83.

20. PRADIER A., LECROISEY F., GAUTHIER J., 1995. A sector study: the goat meat commodity chain in Zimbabwe with special emphasis on Masvingo. Masvingo, Zimbabwe, CIRAD-EMVT/Agritex, 64 p. (Rapport interne)

21. SO UFFLET JF., 1988. La filière bétail et viande bovine : fonctionnement et évolution de 1960 à 1985, perspectives 1990. Livre 1. Thèse doct. Etat Sci. écon., U niversité Montpellier I, Montpellier, France, $170 \mathrm{p}$.

Reçu le 5.3.97, accepté le 14.8.97

\section{Resumen}

Gauthier J., Pradier A., Lecroisey F., Shumba C. Estudio de la filial caprina en Zimbabwe

Este artículo presenta un estudio del sector caprino en Zimbabwe, particularmente en la provincia de Masvingo, basado sobre el uso del concepto de filial como instrumento de análisis. Después de una breve justificación de la elección del análisis y de una presentación de los instrumentos de metodología escogidos, se proponen dos representaciones de la filial. El segundo modelo, más importante para entender las formas de funcionamiento de esta filial, se comenta seguidamente en referencia a los datos de contabilidad. Se deduce que este tipo de análisis permite identificar los interlocutores privilegiados para el establecimiento de acciones de desarrollo, orientado hacia el enfoque de los circuitos de comercialización de la carne de cabra y del mejoramiento de los ingresos de los pequeños productores.

Palabras clave: Carne de cabra - Corriente de mercadeo Economía agrícola - Modelo - Zimbabwe. 